\title{
Oropharyngeal Colonization by Streptococcus pneumoniae among Medical Students in Indonesia
}

\author{
Stella Valencia, ${ }^{1}$ Yanti Mulyana, ${ }^{2}$ Diah Dhianawaty ${ }^{3}$ \\ ${ }^{1}$ Faculty of Medicine Universitas Padjadjaran, ${ }^{2}$ Department of Microbiology and Parasitology \\ Faculty of Medicine Universitas Padjadjaran, ${ }^{3}$ Department of Biochemistry and Molecular Biology \\ Faculty of Medicine Universitas Padjadjaran
}

\begin{abstract}
Background: Streptococcus pneumoniae may colonize the upper respiratory tract without causing any symptoms. Medical students may be inhabited by these bacteria and transmit them to patients who were prone to infections. Streptococcus pneumoniae resistance to antibiotics was recently reported. This study was conducted to determine whether there was Streptococcus pneumoniae colonization among Medical Students of the Faculty of Medicine Universitas Padjadjaran Batch 2011 and analyze its susceptibility patterns towards several antibiotics.

Methods: A descriptive study was conducted involving 75 Medical Students of the Faculty of Medicine Universitas Padjadjaran Batch 2011 that met the selection criteria. After informed consent, oropharyngeal throat swab was taken and further identification was carried out. Once Streptococcus pneumoniae colony was identified, susceptibility testing would be performed.

Results: The identification results indicate that 7 students (9\%) were colonized by Streptococcus pneumoniae. The susceptibility test showed that out of 7 isolates, 2 were resistant to 1 antibiotic, 1 was resistant to 2 antibiotics, and 4 were resistant to 3 antibiotics. Meanwhile, Streptococcus pneumoniae was resistant to trimethoprim-sulfamethoxazole (71\%), oxacillin (71\%), erythromycin (57\%), and levofloxacin (14\%).

Conclusions: Streptococcus pneumoniae colonization is found among medical students. All Streptococcus pneumoniae are resistant to one or more antibiotics, mostly to trimethoprim-sulfamethoxazole and oxacillin. [AMJ.2016;3(3):349-54]
\end{abstract}

Keywords: Medical student, Streptococcus pneumoniae, susceptibility pattern

\section{Introduction}

Streptococcus pneumoniae infection results in various diseases from the mild ones to the life-threatening diseases such as bacteremic pneumonia and meningitis, thus making this infection an important cause of morbidity and mortality worldwide. ${ }^{1}$ Addition to causing diseases, these bacteria may colonize the human upper respiratory tract without causing any symptoms. ${ }^{2}$ A healthy human being that has this bacteria colonization without causing any symptoms is called a carrier. In developing countries, the prevalence of Streptococcus pneumoniae carriers could reach 70-90\% in children under five years old and more than $40 \%$ in adults. Carriers play a major role in the spread of those bacteria through droplets when sneezing or coughing. ${ }^{1}$ Medical students can also be exposed to Streptococcus pneumoniae, become a carrier, and transmit those bacteria to others, particularly patients who are prone to infections. In early 2015, Medical Students of the Faculty of Medicine Universitas Padjadjaran Batch 2011 enrolled in the Program Studi Profesi Dokter (PSPD) at Dr. Hasan Sadikin General Hospital in Bandung. Their enrollment to the hospital environment would play a role in the Streptococcus pneumoniae transmission at the hospital. This bacterial infection can be treated by antibiotics, but its resistance to antibiotics was recently reported. ${ }^{3}$

Based on the explanation above, it is considered necessary to conduct a study to determine whether there was Streptococcus pneumoniae colonization among Medical Students of the Faculty of Medicine Universitas Padjadjaran Batch 2011 and identify its

Correspondence: Stella Valencia, Faculty of Medicine, Universitas Padjadjaran, Jalan Raya Bandung-Sumedang Km.21, Jatinangor, Sumedang, Indonesia, Phone: +62 81394133310 Email: valenz_stell@yahoo.co.id 
susceptibility patterns toward several antibiotics.

\section{Methods}

This descriptive study was conducted at the Microbiology Laboratory, Faculty of Medicine, Universitas Padjadjaran from September to October 2014 after an approval from the Health Research Ethics Committee of the Faculty of Medicine, Universitas Padjadjaran. Population of this study was all Medical Students of the Faculty of Medicine Universitas Padjadjaran Batch 2011. Based on the sample formula for a descriptive study with dichotomous variables, the minimum sample size required was 62 persons. The sample size was set to 75 persons to compensate dropped out participants. Participants were chosen by using the simple random sampling method from the population that met the selection criteria, namely the inclusion and exclusion criteria. The inclusion criterion was Medical Students of the Faculty of Medicine Universitas Padjadjaran Batch 2011 who were studying in the fourth year during the study, while the exclusion criteria were medical students who refused to participate; were not available when the study was held; had been suffering from respiratory tract infections during the study; had been treated by antibiotics in the last 2 weeks prior

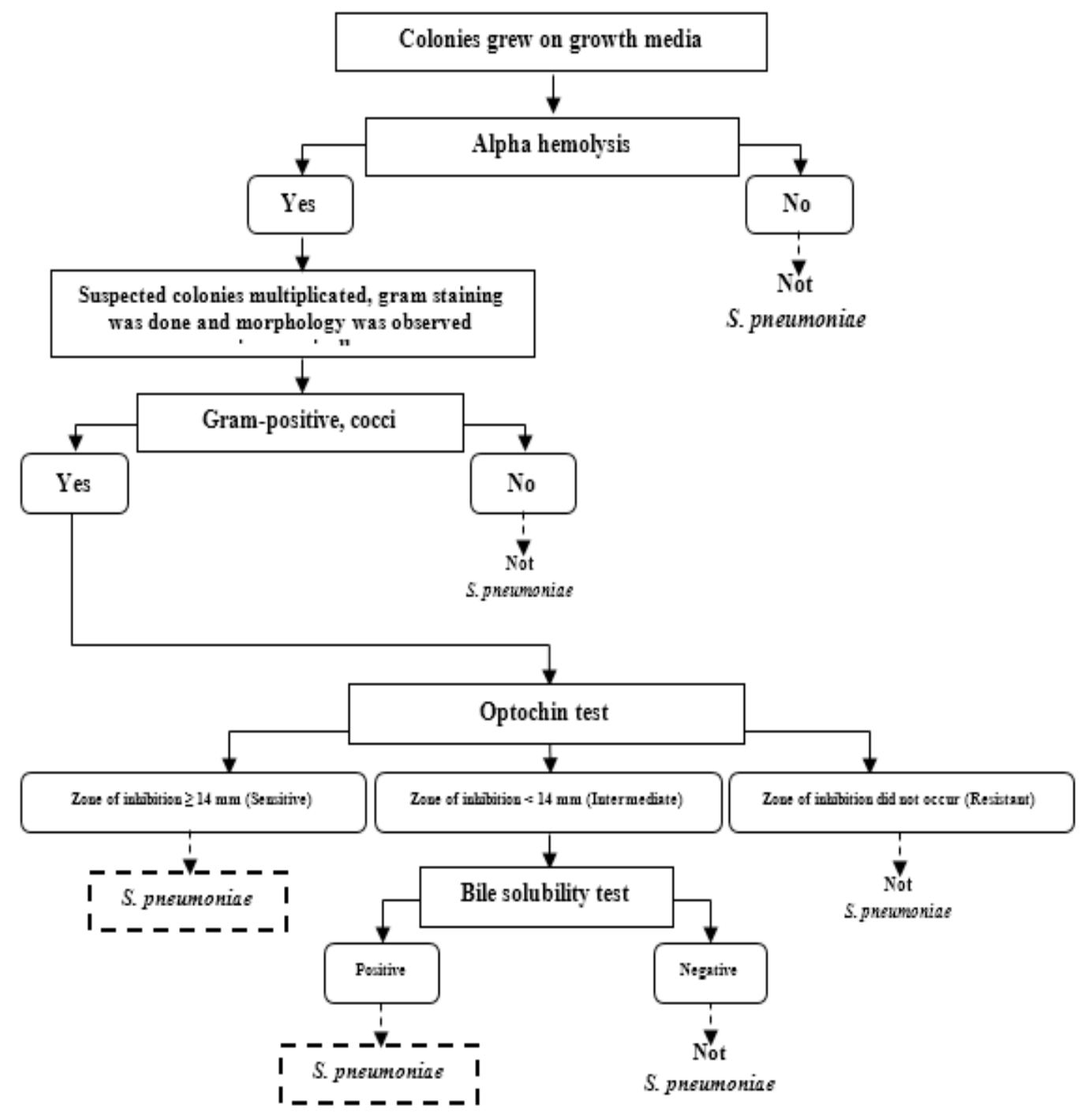

Figure 1 Streptococcus pneumoniae Identification Procedures 
Table 1 Streptococcus pneumoniae colonization

\begin{tabular}{lcc}
\hline \multicolumn{1}{c}{ S. pneumoniae Colonization } & $\mathbf{n}$ & $\mathbf{\%}$ \\
\hline Positive & 7 & 9 \\
Negative & 68 & 91 \\
Total & 75 & 100 \\
\hline
\end{tabular}

to specimens collections. Participants were given explanations about the aim of the study and its procedures, then asked to declare their agreement by signing the informed consent sheet. Once the participant agreed to participate, a throat swab from the oropharynx was performed. The specimens were obtained using a sterile cotton swab that was passed via the mouth without touching any surfaces then swabbed over the oropharyngeal tonsils and posterior wall.

Furthermore, those specimens were directly inoculated in 5\% sheep blood agar with gentamycin to inhibit the growth of normal flora that can also be found in the throat swab. Candle jars were used in order to create an anaerobic ambience (3-5\% CO2). The growth media that had been inoculated and put into candle jars were incubated for 24-48 hours at $\pm 37^{\circ} \mathrm{C}$. After this procedure was conducted, identification of colonies that grew on the growth media which was suspected as the Streptococcus pneumoniae colony underwent the following steps as shown in Figure $1 .^{2}$

The Streptococcus pneumoniae colonization was interpreted as positive if on the growth media at least one suspected colony of Streptococcus pneumoniae was discovered which met all the following criteria: alpha hemolysis; gram-positive cocci; and optochin test results were sensitive or intermediate with positive results in the bile solubility test. When the colonies were not alpha hemolysis; or were not a gram-positive cocci; or optochin test results were resistant or intermediate with a negative result in the bile solubility test, the colonization of Streptococcus pneumoniae were interpreted as negative results. ${ }^{2}$

Colonies that were identified as Streptococcus pneumoniae enrolled in antimicrobial susceptibility testing towards four different antibiotics from different classes which were widely used to treat Streptococcus pneumoniae infections, were: oxacillin (to assess beta-lactams and cephalosporins), erythromycin, trimethoprimsulfamethoxazole, and levofloxacin., ${ }^{2,4}$ The susceptibility testing in this study followed the disk diffusion (Kirby-Bauer) method which was assessed by zone of inhibition diameter formed by each antibiotic disc. ${ }^{2}$ The results were interpreted as Resistant, Intermediate or Sensitive based on the Clinical and Laboratory Standards Institute (CLSI). ${ }^{4}$

Finally, the collected data were processed and presented by frequency distribution and percentage shown in tables.

\section{Results}

All participants that have been given an

Table 2 Antimicrobial Susceptibility Testing

\begin{tabular}{cccccccc}
\hline \multirow{2}{*}{ No. } & \multicolumn{4}{c}{ Susceptibility Testing } & \multicolumn{3}{c}{ Total } \\
\cline { 2 - 8 } & OX & E & SXT & LEV & R & I & S \\
\hline 1 & $\mathrm{R}$ & $\mathrm{S}$ & $\mathrm{R}$ & $\mathrm{S}$ & 2 & 0 & 2 \\
2 & $\mathrm{R}$ & $\mathrm{R}$ & $\mathrm{R}$ & $\mathrm{S}$ & 3 & 0 & 1 \\
3 & $\mathrm{~S}$ & $\mathrm{R}$ & $\mathrm{I}$ & $\mathrm{R}$ & 2 & 1 & 1 \\
4 & $\mathrm{R}$ & $\mathrm{R}$ & $\mathrm{R}$ & $\mathrm{S}$ & 3 & 0 & 1 \\
5 & $\mathrm{~S}$ & $\mathrm{~S}$ & $\mathrm{R}$ & $\mathrm{S}$ & 1 & 0 & 3 \\
6 & $\mathrm{R}$ & $\mathrm{R}$ & $\mathrm{R}$ & $\mathrm{S}$ & 3 & 0 & 1 \\
7 & $\mathrm{R}$ & $\mathrm{S}$ & $\mathrm{S}$ & $\mathrm{S}$ & 1 & 0 & 3 \\
\hline Note: & OX=oxacillin, & E=erythromycin, & SXT=trimethoprim-sulfamethoxazole, LEV=levofloxacin. & R=resistant,
\end{tabular}

I=intermediate, $\mathrm{S}=$ sensitive 
Table 3 Percentage of Antimicrobial Susceptibility Testing

\begin{tabular}{ccccccc}
\hline \multirow{2}{*}{ Antibiotic Discs } & \multicolumn{2}{c}{ Resistant } & \multicolumn{2}{c}{ Intermediate } & \multicolumn{2}{c}{ Sensitive } \\
\cline { 2 - 7 } & $\mathbf{N}$ & $\mathbf{0}$ & $\mathbf{N}$ & $\mathbf{\%}$ & $\mathbf{N}$ & $\mathbf{\%}$ \\
\hline Oxacillin & 5 & 71 & 0 & 0 & 2 & 29 \\
Erythromycin & 4 & 57 & 0 & 0 & 3 & 43 \\
Trimethoprim- & 5 & 71 & 1 & 14 & 1 & 14 \\
sulfamethoxazole & & & & & & \\
Levofloxacin & 1 & 14 & 0 & 0 & 6 & 86 \\
\hline
\end{tabular}

informed consent were agreed to participate in this study. From 75 medical students, 25 were men and 50 were women with age ranged from 19 to 24 years old. The results of isolation and identification of Streptococcus pneumoniae performed to 75 subjects showed that 7 medical students $(9 \%)$ were colonized by Streptococcus pneumonia (Table 1).

Susceptibility testing was performed towards 7 isolates that were positive for Streptococcus pneumoniae. The results were obtained as showed in Table 2.

No specific pattern was found, such as the resistance pattern that always occurs together in certain antimicrobial agents. From 7 isolates enrolled in susceptibility testing, 2 (29\%) were resistant to 1 antibiotic, $1(14 \%)$ was resistant to 2 antibiotics, while $4(57 \%)$ were resistant to 3 antibiotics.

Streptococcus pneumoniae found in this study was resistant mostly to trimethoprimsulfamethoxazole and oxacillin, followed by erythromycin and levofloxacin (Table 3).

\section{Discussion}

This study showed that $9 \%$ of Medical Students of the Faculty of Medicine Universitas Padjadjaran Batch 2011 were colonized by Streptococcus pneumoniae. This finding was lower compared to Hill et al. ${ }^{5}$ in rural Gambian Village, where Streptococcus pneumoniae carriers were found in more than $60 \%$ adults aged 15-39 years. A study by Reis et al. ${ }^{6}$ towards urban slums population in Brazil found that $16.2 \%$ residents aged over 17 years were colonized by Streptococcus pneumoniae. Additionally, in 2002, Mackenzie et al. ${ }^{7}$ also conducted a survey and discovered that 26\% adults were Streptococcus pneumoniae carriers. Those studies mentioned above obtained specimens from different locations compared to this study. They obtained specimens from nasopharynx rather than oropharynx. $^{5-7}$ The different locations where the specimens was taken could affect the percentage of Streptococcus pneumoniae colonization. This was proven by Watt et al. ${ }^{8}$, the study stated that specimens which were taken from nasopharyngeal swabs were more commonly colonized by Streptococcus pneumoniae $(11.1 \%)$ rather than specimens which were taken from oropharyngeal swabs (5.8\%). Moreover, Odutola et al. ${ }^{9}$ found those bacterial colonies by $76.1 \%$ from nasopharyngeal swabs and $21.3 \%$ from oropharyngeal swabs in infants. Furthermore, a study in Uganda ${ }^{10}$ obtained specimens that were taken from the oropharynx, but on the contrary, their participants were HIV-positive adults with a result that indicate $18 \%$ of them were colonized by Streptococcus pneumoniae. The higher result obtained when compared to this study may be due to HIV-positive people who were more at risk of Streptococcus pneumoniae colonization two times greater than HIV-negative people. ${ }^{11}$

According to some sources, factors such as geographical conditions, smoke exposures, socio-economic conditions, weathers, seasons, ages, population densities, respiratory tract infections that have been suffered previously, and HIV status also can affect the incidence of carrier. ${ }^{6,7,12}$ Other reason for low percentage of Streptococcus pneumoniae colonization in medical students was that they were more concerned about hygiene and health rather than the community in general.

Decreased in sensitivity found to at least one antibiotic was observed by susceptibility testing performed to 7 isolates that were colonized by Streptococcus pneumoniae, even 5 isolates $(71 \%)$ were resistant to 2 antibiotics and more (multi drug resistance). This findings were higher than the study conducted in Gambia ${ }^{5}$, which only reached $26.3 \%$. The high number of resistance in our study might be due to the participants who were already exposed to the resistant strains 
of Streptococcus pneumoniae. Other possible causes may be an irrational use of antibiotics such as: people could obtain antibiotics easily without a prescription, patients noncompliance in completing a series of antibiotic treatment, or unwise prescribing that has been done by physicians or pharmacists.13 This irrational use of antibiotics commonly occurred in Indonesia.

The antimicrobial susceptibility testing results showed resistance to trimethoprimsulfamethoxazole, oxacillin, erythromycin, and levofloxacin with percentage 71\%, 71\%, 57\%, and $14 \%$ respectively. Meanwhile, a study conducted by Oteo et al. ${ }^{3}$ stated Streptococcus pneumoniae resistance were $14.1 \%$ to oxacillin, $26.6 \%$ to erythromycin and $0.4 \%$ to levofloxacin. In a study performed by Reis et al. ${ }^{6}$, Streptococcus pneumoniae resistance were $28 \%$ toward trimethoprim-sulfamethoxazole, $22 \%$ toward oxacillin and $2 \%$ toward erythromycin. Bacterial susceptibility patterns to antibiotics were differentin each areaand this can be caused by the difference of antibiotics usage patterns in every different locations. ${ }^{1}$ While decreased in Streptococcus pneumoniae sensitivity to certain antibiotics may be due to the common use of those antibiotics in infections treatment. ${ }^{2}$ High resistance against trimethoprim-sulfamethoxazole in this study may be due to the widely used of trimethoprimsulfamethoxazole as a first choice of antibiotics to treat pneumoniae in children under five years old. Moreover, Streptococcus pneumoniae that was resistant to optochin might be found in this study but their presence were not detected by the identification procedures that have been performed. Nunes et al. ${ }^{14}$ discovered that Streptococcus pneumoniae isolated was resistant to optochin in healthy children.

The specimens of this study were not taken from the nasopharynx but from the oropharynx due to the limitations of the author's competence, because the nasopharynx specimen's collections are more invasive. Even though oxacillin is not used for treatment of human infections, this antibiotic was used in the study because of its benefit in the assessment of Streptococcus pneumoniae sensitivity toward beta-lactams and cephalosporins. If oxacillin resistant isolates were found, further assessment should be conducted to determine Streptococcus pneumoniae sensitivity toward each antibiotics from each class, mainly those which were commonly used for Streptococcus pneumoniae infections treatment. ${ }^{4}$ However, because of time and funding limitations, it was not performed.
A further study with larger scales should be carried out to identify the most common Streptococcus pneumoniae serotypes in Indonesia or a study about bacterial susceptibility patterns toward more types of antibiotics so that more comprehensive patterns would be obtained. Furthermore, it is important to perform transmission control, such as promoting the use of masks among health care workers when dealing with patients who are susceptible to infection and also promote pneumococcal vaccination as a basic immunization for children, since it has been associated with decreased of the adults Streptococcus pneumoniae carriage indirectly. ${ }^{15}$ Rational use of antibiotics should also be improved, either rational antibiotics prescription by physicians or pharmacies, as well as its regulation by government.

From this study, it can be concluded that Streptococcus pneumoniae colonization is found among Medical Students of the Faculty of Medicine Universitas Padjadjaran Batch 2011. All Streptococcus pneumoniae found are resistant to one or more antibiotics, mostly to trimethoprim-sulfamethoxazole and oxacillin.

\section{References}

1. Longo DL, Fauci AS, Kasper DL, Hauser SL, Jameson JL, Loscalzo J, editors. Harrison's principles of internal medicine. $18^{\text {th }}$ ed. New York: McGraw-Hill Education; 2012.

2. Forbes BA, Sahm DF, Weissfeld AS. Bailey \& Scott's diagnostic microbiology. $12^{\text {th }}$ ed. St. Louis: Elsevier Science Health Science Division; 2007.

3. Oteo J, Lázaro E, Abajo FJd, Baquero F, Campos J. Trends in antimicrobial resistance in 1,968 invasive Streptococcus pneumoniae strains isolated in Spanish Hospitals (2001 to 2003): decreasing penicillin resistance in children's isolates. J Clin Microbiol. 2004;42(12):5571-7.

4. CLSI. Performance standards for antimicrobial susceptibility testing; twenty-fourth informational supplement. CLSI document M100-S24. Wayne, PA: Clinical and Laboratory Standards Institute; 2014.

5. Hill PC, Akisanya A, Sankareh K, Cheung YB, Saaka M, Lahai G, et al. Nasopharyngeal carriage of Streptococcus pneumoniae in Gambian villagers. Clin Infect Dis. 2006;43(6):673-9.

6. Reis JN, Palma T, Ribeiro GS, Pinheiro RM, Ribeiro CT, Cordeiro SM, et al. Transmission of Streptococcus pneumoniae in an urban 
slum community. J Infect. 2008;57(3):20413.

7. Mackenzie GA, Leach AJ, Carapetis JR, Fisher J, Morris PS. Epidemiology of nasopharyngeal carriage of respiratory bacterial pathogens in children and adults: cross-sectional surveys in a population with high rates of pneumococcal disease. BMC Infect Dis. 2010;10(1):304.

8. Watt JP, O'brien KL, Katz S, Bronsdon MA, Elliott J, Dallas J, et al. Nasopharyngeal versus oropharyngeal sampling for detection of pneumococcal carriage in adults. JClin Microbiol. 2004;42(11):49746.

9. Odutola A, Antonio M, Owolabi O, Bojang A, Foster-Nyarko E, Donkor S, et al. Comparison of the prevalence of common bacterial pathogens in the oropharynx and nasopharynx of Gambian infants. PLoS One. 2013;8(9):e75558.

10. Blossom DB, Namayanja-Kaye G, NankyaMutyoba J, Mukasa JB, Bakka H, Rwambuya $\mathrm{S}$, et al. Oropharyngeal colonization by Streptococcus pneumoniae among HIVinfected adults in Uganda: assessing prevalenceandantimicrobialsusceptibility. Int J Infect Dis. 2006;10(6):458-64.

11. Gill C, Mwanakasale V, Fox M, Chilengi R,
Tembo M, Nsofwa M, et al. Impact of human immunodeficiency virus infection on Streptococcus pneumoniae colonization and seroepidemiology among Zambian women. J Infect Dis. 2008;197(7):1000-5.

12. Adegbola RA, DeAntonio R, Hill PC, Roca A, Usuf E, Hoet B, et al. Carriage of Streptococcus pneumoniae and other respiratory bacterial pathogens in low and lower-middle income countries: a systematic review and meta-analysis. PLoS One. 2014;9(8):e103293.

13. Sharma R, Sharma CL, Kapoor B. Antibacterial resistance: current problems and possible solutions. Indian J Med Sci. 2005;59(3):120-9.

14. Nunes S, Sá-Leão R, Lencastre $\mathrm{Hd}$. Optochin resistance among Streptococcus pneumoniae strains colonizing healthy children in Portugal. J Clin Microbiol. 2008;46(1):321-4.

15. Hammitt LL, Bruden DL, Butler JC, Baggett HC, Hurlburt DA, Reasonover A, et al. Indirect effect of conjugate vaccine on adult carriage of Streptococcus pneumoniae: an explanation of trends in invasive pneumococcal disease. J Infect Dis. 2006;193(11):1487-94. 